\title{
ELECTROENCEPHALOGRAMS MULTISCALE ENTROPY ANALYSIS OF ALZHEIMER'S DISEASE PATIENTS
}

\author{
J. Escudero ${ }^{1}$, D. Abásolo ${ }^{1}$, R. Hornero ${ }^{1}$, P. Espino $^{2}$ and M. López ${ }^{1}$ \\ ${ }^{1}$ E.T.S. Ingenieros de Telecomunicación, University of Valladolid, Valladolid, Spain \\ ${ }^{2}$ Hospital Clínico San Carlos, Madrid, Spain \\ danaba@tel.uva.es
}

\begin{abstract}
This paper aims to analyse the electroencephalogram (EEG) background activity of Alzheimer's disease (AD) patients using the multiscale entropy (MSE) method. The MSE has been recently introduced as a measure of the signal complexity that inspects different time scales. EEGs were recorded from 19 scalp electrodes in 8 AD patients and 8 control subjects and the MSE analysis was performed on each artefact-free EEG epoch. The MSE analysis of the data suggests a high degree of complexity and the presence of long time correlations in the EEG. Moreover, we found significant differences ( $p$-value $<0.01$, Student's $t$-test) between the MSE profile of control subjects and $\mathrm{AD}$ patients at 7 electrodes (Fp1, Fp2, T5, P3, P4, O1 and $\mathrm{O} 2$ ). We can infer from these findings that the $M S E$ may be a useful tool to inspect the complexity of the $\mathrm{EEG}$ and, therefore, increase our knowledge of $\mathrm{AD}$.
\end{abstract}

Keywords: Alzheimer's disease, electroencephalogram, multiscale entropy, complexity, time scales.

\section{INTRODUCTION}

Alzheimer's disease (AD) is a very frequent disorder among elderly population and it is considered the most frequent cause of dementia in western countries [1]. AD causes progressive cognitive and intellectual deficits and behaviour disturbance [1]. Physiologically, AD is characterized by widespread neuronal cell loss and the appearance of senile plaques and neurofibrillary tangles [1]. Methods of medical imaging are used for diagnosis in medium stages of the disease. In addition, mental status test, such as the Folstein's Mini Mental State Examination (MMSE) [2], are frequently used to determine the severity of the dementia. However, the diagnosis can only be confirmed by necropsy. The usefulness of the electroencephalogram (EEG) as a diagnostic tool for AD and other kinds of dementia has been investigated for several decades [3].

The EEG signals have been extensively analysed by linear and non-linear methods [3]. For several decades, the classical linear techniques were the only available tools to study the EEG. However, the non-linear behaviour of the neurons and the ability of the brain to perform sophisticated cognitive tasks support the hypothesis that the brain may not be a simple stochastic system [4]. Based on this hypothesis, new advanced statistical methods (such as EEG filtering based on blind source separation [5]) and non-linear techniques derived from the chaos theory (e.g., as the correlation dimension
$\left(D_{2}\right)$ [6] and the first Lyapunov exponent $\left(L_{1}\right)$ [7]) have been used in order to characterize pathologic dynamics in EEG activity. $D_{2}$ and $L_{1}$ were the first non-linear analysis techniques applied to EEGs. Several studies have shown that they can provide potentially useful diagnostic information from EEG of patients with different mental diseases [3, 8]. However, the application of $D_{2}$ or $L_{1}$ on biomedical signals is difficult to justify from a theoretical point of view because these methods require a large amount of stationary data, something that is almost impossible to achieve when working with biomedical recordings [9]. Therefore, it becomes necessary to analyse the EEG by means of other non-linear methods. While some of these methods are based on measuring the "regularity" of the data [10], other algorithms (e.g., the wavelet complexity [11] or the LZ complexity [4]) are based on the concept of "complexity", which can sometimes be confusing.

Complex systems are neither absolutely regular nor absolutely random [11, 12]. Thus, a new complexity estimator has been recently proposed: the multiscale entropy $(M S E)[12,13]$. The $M S E$ focuses on estimating the complexity of a signal by determining the information expressed on multiple time scales, and it has been already used to analyse biological data [12].

In this pilot study the MSE has been used to examine the EEG background activity of AD patients and agematched control subjects. We wanted to test the hypothesis that the MSE analysis of EEG background activity might be useful to differentiate $\mathrm{AD}$ patients from control subjects.

\section{MATERIALS AND METHODS}

\section{Subjects}

Eight $\mathrm{AD}$ patients $($ age $=75.9 \pm 3.6$ years, mean \pm standard deviation (SD)), fulfilling the criteria of probable $\mathrm{AD}$, and 8 control subjects $(75.1 \pm 5.5$ years, mean $\pm \mathrm{SD}$ ) participated in this study. The AD patients were recruited from Alzheimer's Patients' Relatives Association of Valladolid (AFAVA). The EEG was registered in the University Hospital of Valladolid (Spain) after all the subjects had undergone a meticulous clinical evaluation. The mean MMSE [2] score for the $\mathrm{AD}$ group was $14.0 \pm 5.9$ points (mean \pm $\mathrm{SD})$, so the average degree of the $\mathrm{AD}$ is moderate. However, three patients had a MMSE score of less than 12 points, and therefore suffered from severe dementia. The 8 age-matched control subjects had not got any past 
or present mental disorder and all their MMSE score values were 30 .

The local ethics committee approved the study. All control subjects and all caregivers of the patients gave their informed consent for participation in this study.

\section{EEG recording}

EEGs were recorded by a Profile Study Room 2.3.411 EEG equipment (Oxford Instruments) at electrodes F3, F4, F7, F8, Fp1, Fp2, T3, T4, T5, T6, C3, C4, P3, P4, $\mathrm{O} 1, \mathrm{O} 2, \mathrm{Fz}, \mathrm{Cz}$ and $\mathrm{Pz}$ of the international 10-20 system. The sample frequency was $256 \mathrm{~Hz}$, with a 12bit A-to-D precision. During the EEG recording, the subjects were asked to remain relaxed, with closed eyes and awake to reduce the presence of artefacts. At least 5 minutes of EEG data were recorded from each subject.

The recordings were visually inspected by a specialist physician to select data with minimal artefact activity. Artefact-free epochs of 5 s (1280 points) were selected and copied to ASCII files for off-line analysis. Before the non-linear analysis was carried out, the data were processed with a bandpass filter with cut-off frequencies at $0.5 \mathrm{~Hz}$ and $40 \mathrm{~Hz}$.

\section{Multiscale entropy (MSE) analysis}

The MSE was introduced as a tool to estimate the complexity of signals by evaluating their orderliness on different time scales [13]. In order to represent the system dynamics on such time scales, coarse-grained versions of the original signal are built. The regularity of these coarse-grained sequences is assessed by the sample entropy (SampEn) [14], which is based on the approximate entropy [15]. The use of the SampEn provides important advantages: it is independent of the signal length and model, and it can be applied to relatively short, noisy data sets [14].

Formally, given a one-dimensional discrete time series, $\left\{x_{1}, \ldots, x_{i}, \ldots, x_{L}\right\}$, first we must build successive coarse-grained series, $\left\{y^{(\tau)}\right\}$, corresponding to the scale factor, $\tau$. In order to make the coarse-grained sequences, we have to divide the original series into nonoverlapping windows of length $\tau$. Then, we average the values of the data points inside each window. The process [12] is summarized in (1):

$$
y_{j}^{(\tau)}=\frac{1}{\tau} \sum_{i=(j-1) \tau+1}^{j \tau} x_{i}, \quad 1 \leq j \leq \frac{L}{\tau}=L^{(\tau)} .
$$

Once such coarse-grained time series are built, we estimate their SampEn [12]. Briefly, SampEn measures the logarithmic conditional probability that sets of patterns which are closer than a tolerance, $r$, for a pattern length, $m$, remain similar at the next point (pattern length $m+1$ ), where self-matches are not included in calculating the probability. Larger SampEn values correspond to more irregular signals [14]. Given a sequence $\{y(n)\}=y(1), y(2), \ldots, y(N)$, the SampEn is computed as follows [14]:
1. Form $m$ vectors $Y_{m}(1), \ldots, Y_{m}(N-m+1)$ defined by $Y_{m}(i)=[y(i), \ldots, y(i+m-1)]$, for $1 \leq i \leq N-m+1$. Such vectors, which begin with the $i$ th point, represent $m$ consecutive $y$ values.

2. Define the distance between vectors $Y_{m}(i)$ and $Y_{m}(j)$, $d\left[Y_{m}(i), Y_{m}(j)\right]$, as the maximum norm:

$$
d\left[Y_{m}(i), Y_{m}(j)\right]=\max _{k=0, \ldots, m-1}(|y(i+k)-y(j+k)|) .
$$

3. For a given $Y_{m}(i)$, count the number of $j(1 \leq j \leq N$ $m, j \neq i$ ), denoted as $B_{i}$, such that the distance between $Y_{m}(i)$ and $Y_{m}(j)$ is less than or equal to $r$. Then, for $1 \leq i \leq N-m$,

$$
B_{i}^{m}(r)=\frac{B_{i}}{(N-m-1)} .
$$

4. Define $B^{m}(r)$ as

$$
B_{m}(r)=\frac{1}{(N-m)} \sum_{i=1}^{N-m} B_{i}^{m}(r)
$$

5. Increase the dimension to $m+1$ and calculate $A_{i}$ as the number of $Y_{m+1}(i)$ within $r$ of $Y_{m+1}(j)$, where $j$ ranges from 1 to $N-m(i \neq j)$. Then define

$$
A_{i}^{m}(r)=\frac{A_{i}}{(N-m-1)} .
$$

6. Set $A^{m}(r)$ as

$$
A_{m}(r)=\frac{1}{(N-m)} \sum_{i=1}^{N-m} A_{i}^{m}(r)
$$

The SampEn is estimated by the statistic

$$
\operatorname{SampEn}(m, r, N)=-\ln \left(\frac{A^{m}(r)}{B^{m}(r)}\right) \text {. }
$$

Comparisons between series can only be done with values of $m, r$ and $N$ unchanged [14]. Following the guides given in $[14,16]$, we fixed $m$ and $r$ at the widely used values $m=1$ and $r=0.25$ times the SD of the original series. The largest time scale analysed was $\tau_{M A X}$ $=12$. Hence, the shortest coarse-grained sequence built has more than 100 points. This length is able to produce good statistical reproducibility with the SampEn [14].

The representation of the SampEn values versus the scale provides curves with useful information about the analysed signal [12]. In this study, we used Student's $t$ test to evaluate statistical differences between the MSE profiles of $\mathrm{AD}$ patients and control subjects.

\section{RESULTS}

The MSE analysis was performed for channels F3, F4, F7, F8, Fp1, Fp2, T3, T4, T5, T6, C3, C4, P3, P4, O1 and $\mathrm{O} 2$ with $m=1$ and $r=0.25$ times the SD of the original time series. After a visual inspection of the $M S E$ profiles was performed, we could see that it is usually possible to divide the $M S E$ profiles into two parts. The first one, for $\tau \leq 5$, approximately, presents an increasing slope, whereas the values of the second one, for $6 \leq \tau$, are almost constant or have a slight decreasing slope. Based on this limit, we characterized every data 


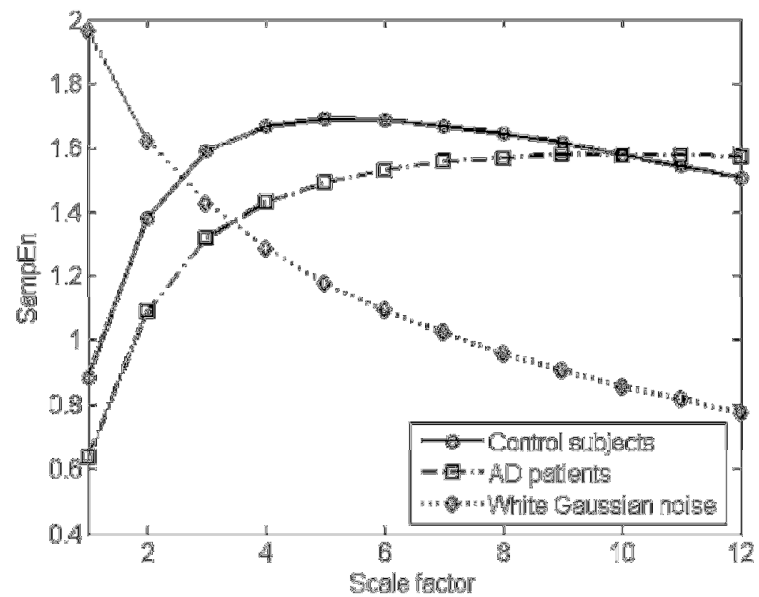

Fig. $1 M S E$ analysis of the 8 control subjects and 8 AD patients with $m=1$ and $r=0.25$ times the SD of the original time series at electrode $\mathrm{O} 1$. The mean $M S E$ profile of 500 white Gaussian noise series also composed of 1280 points is shown for comparison.

epoch by the slope of the MSE profile for $\tau \leq 5$ (small time scales, $t \leq 20 \mathrm{~ms}$ ) and for $\tau \geq 6$ (large time scales, $t$ $\geq 23 \mathrm{~ms}$ ).

The MSE curves are characterized by a steep slope on the smaller time scales, while the slope on the larger time scales is much smoother. However, there are important differences between the MSE profiles of both groups. In the control group, the irregularity of the coarse-grained sequences decreases on the larger time scales. On the other hand, the coarse-grained sequences of the AD patients are usually slightly more irregular as we analyse larger time scales. Furthermore, the values of SampEn are higher for control subjects than for AD patients, except for the largest time scales. We can infer that the control subjects have a more complex EEG background activity than the AD patients [12]. Fig. 1 shows the MSE profile at electrode $\mathrm{O} 1$ for both groups. The average MSE profiles at other electrodes are similar to these. For comparison, fig. 1 also depicts the mean $M S E$ analysis of 500 white Gaussian noise series.

The average slope values (Mean $\pm \mathrm{SD}$ ) of the $M S E$ profiles for small and large time scales are summarized in table 1 and table 2, respectively. Student's $t$-test was used to determine whether there are significant differences between both subject groups. Significant differences were found at 7 electrodes for the slope on the large time scales $(\tau \geq 6)$.

\section{DISCUSSION AND CONCLUSIONS}

In this preliminary study we have applied the $M S E$ to analyse the EEG background activity of 8 AD patients and 8 age-matched control subjects. The MSE is a new method to assess the complexity of a signal [12]. It is based on estimating the orderliness, by means of the SampEn, of several coarse-grained versions of the original signal. These coarse-grained sequences represent the system on different time scales. The MSE

\begin{tabular}{c|ccc} 
Channel & $\begin{array}{c}\text { AD patients } \\
\text { (Mean } \pm \text { SD) }\end{array}$ & $\begin{array}{c}\text { Control subjects } \\
\text { (Mean } \pm \text { SD) }\end{array}$ & $\begin{array}{c}t \text {-test } \\
\text {-value }\end{array}$ \\
\hline F3 & $0.1930 \pm 0.0319$ & $0.1859 \pm 0.0326$ & 0.6695 \\
F4 & $0.1952 \pm 0.0244$ & $0.1875 \pm 0.0220$ & 0.5199 \\
F7 & $0.1659 \pm 0.0313$ & $0.1904 \pm 0.0267$ & 0.1142 \\
F8 & $0.1731 \pm 0.0339$ & $0.1915 \pm 0.0286$ & 0.2620 \\
Fp1 & $0.1540 \pm 0.0424$ & $0.1818 \pm 0.0277$ & 0.1429 \\
Fp2 & $0.1712 \pm 0.0244$ & $0.1821 \pm 0.0298$ & 0.4366 \\
T3 & $0.1536 \pm 0.0431$ & $0.1709 \pm 0.0363$ & 0.3986 \\
T4 & $0.1369 \pm 0.0581$ & $0.1780 \pm 0.0321$ & 0.1018 \\
T5 & $0.2018 \pm 0.0195$ & $0.2009 \pm 0.0377$ & 0.9535 \\
T6 & $0.1984 \pm 0.0289$ & $0.2028 \pm 0.0311$ & 0.7761 \\
C3 & $0.1929 \pm 0.0272$ & $0.1968 \pm 0.0282$ & 0.7847 \\
C4 & $0.1928 \pm 0.0350$ & $0.2037 \pm 0.0270$ & 0.4982 \\
P3 & $0.2148 \pm 0.0168$ & $0.2143 \pm 0.0288$ & 0.9619 \\
P4 & $0.2223 \pm 0.0173$ & $0.2167 \pm 0.0323$ & 0.6694 \\
O1 & $0.2021 \pm 0.0267$ & $0.1916 \pm 0.0420$ & 0.5622 \\
O2 & $0.2040 \pm 0.0273$ & $0.1900 \pm 0.0360$ & 0.3975
\end{tabular}

Table 1: average slope values of the $M S E$ profiles on small time scales $(\tau \leq 5)$ of the EEGs for both groups with $m=1$ and $r=0.25$ times the SD of the original series.

\begin{tabular}{c|ccc} 
Channel & $\begin{array}{c}\text { AD patients } \\
(\text { Mean } \pm \text { SD) }\end{array}$ & $\begin{array}{c}\text { Control subjects } \\
(\text { Mean } \pm \text { SD })\end{array}$ & $\begin{array}{c}t \text {-test } \\
p \text { value }\end{array}$ \\
\hline F3 & $0.0077 \pm 0.0163$ & $-0.0167 \pm 0.0186$ & 0.0141 \\
F4 & $0.0042 \pm 0.0166$ & $-0.0167 \pm 0.0183$ & 0.0314 \\
F7 & $0.0066 \pm 0.0130$ & $-0.0107 \pm 0.0194$ & 0.0540 \\
F8 & $-0.0010 \pm 0.0235$ & $-0.0157 \pm 0.0116$ & 0.1322 \\
Fp1* & $0.0068 \pm 0.0110$ & $-0.0174 \pm 0.0150$ & 0.0025 \\
Fp2* & $0.0107 \pm 0.0115$ & $-0.0195 \pm 0.0147$ & 0.0004 \\
T3 & $-0.0072 \pm 0.0247$ & $-0.0224 \pm 0.0271$ & 0.2604 \\
T4 & $-0.0151 \pm 0.0387$ & $-0.0296 \pm 0.0207$ & 0.3679 \\
T5* & $0.0129 \pm 0.0222$ & $-0.0250 \pm 0.0220$ & 0.0040 \\
T6 & $0.0079 \pm 0.0237$ & $-0.0235 \pm 0.0193$ & 0.0114 \\
C3 & $-0.0020 \pm 0.0166$ & $-0.0269 \pm 0.0240$ & 0.0308 \\
C4 & $-0.0048 \pm 0.0230$ & $-0.0287 \pm 0.0259$ & 0.0717 \\
P3* & $0.0135 \pm 0.0211$ & $-0.0243 \pm 0.0197$ & 0.0023 \\
P4* & $0.0072 \pm 0.0271$ & $-0.0323 \pm 0.0223$ & 0.0066 \\
O1* & $0.0090 \pm 0.0232$ & $-0.0302 \pm 0.0154$ & 0.0014 \\
O2* & $0.0074 \pm 0.0269$ & $-0.0310 \pm 0.0208$ & 0.0065
\end{tabular}

Table 2: average slope values of the $M S E$ profiles on large time scales $(\tau \geq 6)$ of the EEGs for both groups with $m=1$ and $r=0.25$ times the SD of the original series. Significant group differences are marked with an asterisk.

has some important advantages over other non-linear techniques: it can be applied to relatively noisy time series, irrespective of whether their origin is stochastic or deterministic [12].

We have found that the MSE profiles of the EEG background activity of both subject groups show a typical shape which reveals the complex origin of the EEG [12]. Since the control subjects' EEGs are more irregular than the recordings of the $\mathrm{AD}$ group on almost 
all the time scales, we can infer that the EEG background activity is more complex in the former [12]. This result agrees with other studies that found less complexity or less irregularity in EEG recordings of AD patients than control subjects [3, 8, 10]. The main differences in the shape of the MSE profiles of both groups are on the larger time scales. Moreover, we have found significant differences between both groups on the larger scales at electrodes Fp1, Fp2, T5, P3, P4, O1 and $\mathrm{O} 2$. To be precise, the irregularity of the coarsegrained sequences tends to decrease for the control subjects whereas it remains almost constant or slightly increases for the AD group. This result shows that the correlation in the EEG background activity of the control subjects weakens on the largest time scales [12].

Some limitations of our study merit consideration. First of all, the sample size was small. Although the results indicate that the $M S E$ could be useful in order to help to diagnose $\mathrm{AD}$, the study should be extended on a much larger patient population. Moreover, AD can only be diagnosed without any probability of error by necropsy. Thus, the AD patients' diagnoses are not absolutely reliable, and some patients might be misclassified. Finally, other physiological and pathological states of the brain should be analysed with $M S E$ in order to know whether the findings reported in this study are specific to AD or not.

To sum up, we found significant differences between both subject groups at 7 electrodes. Although this study is preliminary, the MSE analysis of the EEGs has shown that $\mathrm{AD}$ could be characterized not only by changes in the brain activity on the shortest time scale, but also by an abnormal behaviour of the EEG on deeper time scales. Nevertheless, further studies must be carried out to substantiate this suggestion.

\section{ACKNOWLEDGMENTS}

This study was partially supported by a project from the "Consejería de Fomento de la Junta de Castilla y León" and "Ministerio de Educación y Ciencia" and FEDER grant MTM 2005-08519-C02-01.

\section{REFERENCES}

[1] Bird T.D. (2001): 'Alzheimer's disease and other primary dementias' in Harrison's principles of internal medicine, Braunwald E. et al (Eds), The McGraw-Hill Companies Inc, New York, NY, pp. 2391-2399.

[2] Folstein M.F., Folstein S.E. and McHugh P.R. (1975): 'Mini-mental state. A practical method for grading the cognitive state of patients for the clinician', Journal of Psychiatric Research, 12, pp. 189-198.

[3] Jeong J. (2004): 'EEG dynamics in patients with Alzheimer's disease', Clinical Neurophysiology, 115, pp. 1490-1505.
[4] Zhang X.-S., Roy R.J. and Jensen E.W. (2001): 'EEG complexity as a measure of depth of anesthesia for patients', IEEE Transactions on Biomedical Engineering, 48, pp. 1424-1433.

[5] Cichocki A., Shishkin S.L., Musha T., Leonowicz Z., Asada T. and Kurachi T. (2005): 'EEG filtering based on blind source separation (BSS) for early detection of Alzheimer's disease', Clinical Neurophysiology, 116, pp. 729-737.

[6] Grassberger P. and Procaccia I. (1983): 'Measuring the strangeness of strange attractors', Physica D, 9, pp. 189-208.

[7] Wolf A., Swift J.B., Swinney H.L. and Vastano J.A. (1985): 'Determining Lyapunov exponents from a time-series', Physica D, 16, pp. 285-317.

[8] Besthorn C., Sattel H., Geiger-Kabisch C., Zerfass R. and Förstl H. (1995): 'Parameters of EEG dimensional complexity in Alzheimer's disease', Electroencephalography and Clinical Neurophysiology, 95, pp. 84-89.

[9] Eckmann J.P. and Ruelle D. (1992): 'Fundamental limitations for estimating dimensions and Lyapunov exponents in dynamical systems', Physica D, 56, pp. 185-187.

[10] Abásolo D., Hornero R., Espino P., Álvarez D. and Poza J. (2006): 'Entropy analysis of the EEG background activity in Alzheimer's disease patients', Physiological Measurement, 27, pp. 241253.

[11] Rosso O.A., Martin M.T. and Plastino A. (2003): 'Brain electrical activity analysis using waveletbased informational tools (II): Tsallis nonextensivity and complexity measures', Physica A, 320, pp. 497-511.

[12] Costa M., Goldberger A.L. and Peng C.K. (2005): 'Multiscale entropy analysis of biological signals', Physical Review E, 71 (2), 021906.

[13] Costa M., Goldberger A.L. and Peng C.K. (2002): 'Multiscale entropy analysis of complex physiologic time series', Physical Review Letters, 89 (6), 068102.

[14] Richman J.S. and Moorman J.R. (2000): 'Physiological time-series analysis using approximate entropy and sample entropy', American Journal of Physiology (Heart and Circulatory Physiology), 278, pp. H2039-H2049.

[15] Pincus S.M. (1991): 'Approximate entropy as a measure of system complexity', Proceedings of the National Academy of Sciences of the USA, 88, pp. 2297-2301.

[16] Lake D.E., Richman J.S., Griffin M.P. and Moorman J.R. (2002): 'Sample entropy analysis of neonatal heart rate variability', American Journal of Physiology (Regulatory, Integrative and Comparative Physiology), 283, pp. R789-R797. 